Original Article

\title{
Effects of Exercise Therapy on Blood Lipids of Obese Women
}

\author{
Hyo-Cheol Lee, $\mathrm{PhD}^{1)}$, Tag Heo, MD, $\mathrm{PhD}^{2)^{*}}$ \\ 1) Department of Emergency Medical Services, College of Health Science, Honam University, Republic \\ of Korea \\ 2) Department of Emergency Medicine, Chonnam National University College of Medicine, Chonnam \\ National University Hospital: 8 Hak-dong, Dong-gu, Gwangju 500-742, Republic of Korea
}

\begin{abstract}
Purpose] The aim of this study was to examine the effects of exercise therapy on blood lipids of obese women. [Subjects] The subjects were 24 clinically obese women. [Methods] The subjects were divided into an exercise group $(n=12)$, and a control group $(n=12)$. The exercise group performed exercise for 10 weeks with diet restriction. We investigated how this type of exercise influenced blood lipids. Blood was taken before and after the intervention. The collected blood was centrifuged at 3,000 rpm for 10 minutes; and plasma was preserved at $-73^{\circ} \mathrm{C}$. For hemanalysis, a medical laboratory was asked to analyze total cholesterol and triglyceride. The exercise was performed 5 times per week, and an exercise was performed every two days, and exercise program was composed of warm up, main exercise and cool down. [Results] There were significant differences between the two groups in blood lipid levels after the intervention. The differences between the two groups in TC and TG were significant. [Conclusion] TC and TG significantly inproved in the experimental group showing that the exercise was effective at improveing blood lipid levels.

Key words: Blood lipid, Obese, Exercise therapy
\end{abstract}

(This article was submitted Feb. 28, 2014, and was accepted Apr. 28, 2014)

\section{INTRODUCTION}

Regular exercise improves the blood lipids and has a positive effect on endothelial cells of the blood vessel, eventually slowing down or preventing progress of the arteriosclerosis. As therapies for obesity, dietary therapy, exercise therapy, medicinal therapy, and behavior therapy have been used and among then exercise therapy is the most economical, safe, and fundamental treatment of all obesity treatments $^{1)}$. Furthermore, a clinical survey reported that $67.5 \%$ of patients with obesity lacked exercise, and overweight persons were found among those who work for a long time sitting on a chair ${ }^{2}$. These findings demonstrate how physical activity is related to obesity. Dietary therapy is a treatment which controls only calorie intake. It has a big effect on weight-loss in a short period of time, but this can cause several problems, such as decrease in lean body mass (LBM) due to loss of muscular tissues, a decline in the basal metabolic rate (BMR) at rest, and the induction of myofunctional disorder ${ }^{3,4)}$.

Resistance weight training was to have a positive effect on the reduction of body fat by increasing muscle tissues

*Corresponding author. Tag Heo (E-mail: docheo@hanmail. net)

C2014 The Society of Physical Therapy Science. Published by IPEC Inc. This is an open-access article distributed under the terms of the Creative Commons Attribution Non-Commercial No Derivatives (by-ncnd) License $<$ http://creativecommons.org/licenses/by-nc-nd/3.0/>. maintaining of muscular strength, increasing in fat-free mass; and reducing blood lipids and lipoprotein metabolism, emphasizing the importance of resistance weight training as an effective method for weight control. Furthermore, controlling inter-set rest times during weight training significantly affected blood lipid concentrations and body construction $^{4}$.

Weight training also improves the insulin reaction, increases bone density or prevents its loss, and reduces the loss of muscle mass and muscular strength which accompanies aging ${ }^{5}$. In addition, weight training has also been reported to increase muscle strength and the cross-sectional area of muscle not only in men, but also in women in middle and old age. Accordingly, it is desirable that an exercise program for the most safe and efficient weight loss should be composed of a combination aerobic exercise with resistance weight training tailrored to the subject's level. In a recent study, a combination of aerobic exercise and resistance weight training performed by middle-aged women with obesity was found to have a positive effect on body composition and blood lipids ${ }^{6,7)}$. Another study reported the importance of dietary therapy as well as exercise therapy, and stated that exercise therapy without considering dietary therapy would not reduce body fat ${ }^{8)}$.

A few findings have recently been reported regarding the effects of exercise on women with obesity accompanying adult diseases and illnesses. However, they are insufficient and further, more specific and diverse experiments and researches are necessary into how dietary exercises can be combined to create an ideal exercise program tailored to 
1676 J. Phys. Ther. Sci. Vol. 26, No. 11, 2014

Table 1. Characteristics of the subjects

\begin{tabular}{lcccc}
\hline Group & Age (yrs) & Height $(\mathrm{cm})$ & Weight $(\mathrm{kg})$ & (\%fat) \\
\hline Exercise & $51.3 \pm 7.34$ & $152.2 \pm 8.11$ & $65.9 \pm 3.3$ & $34.4 \pm 9.8$ \\
Control & $50.5 \pm 8.41$ & $154.5 \pm 6.33$ & $66.2 \pm 2.6$ & $33.5 \pm 10.2$ \\
\hline
\end{tabular}

personal characteristics. The purpose of this study was to examine the effects of exercise therapy on blood lipids of obese women.

\section{SUBJECTS AND METHODS}

The subjects were 24 women with no habit of regular exercise (Table 1). They were selected using bioelectrical impedance measures which showed they had body fat percentages of over $30 \%$. None of the subjects had high blood pressure or diabetes and none had performed systematic exercise for at least 3 months. The subjects were divided into an group $(\mathrm{n}=12)$, which performed an exercise program for 10 weeks, and a control group $(n=12)$, which performed no exercise. The subjects of the exercise group performed exercise for 10 weeks diet restriction, and we investigated how this type of exercise influenced on blood lipids; consequently aiming at preparing a basic data for designing appropriate and developmental exercise methods and programs to treat obesity to reduce the risk of developing coronary diseases. Blood was taken before and after the intervention by a nurse. The collected blood was centrifuged at $3,000 \mathrm{rpm}$ for 10 minutes and the plasma was preserved at $-73{ }^{\circ} \mathrm{C}$. For hemanalysis, a medical laboratory was asked to analyze total cholesterol and triglyceride. All experiments were reviewed and approved by the Committee of the Honam University. The purpose and procedure of the study were explained to the subjects who voluntarily signed an informed consent agreement before participating in the experiment.

For the exercise load test of this study, all the subjects arrived at the laboratory 40 minutes before the experiment. They performed 10 minutes warm-up and 10 minutes treadmill workout then took a rest of 20 minutes before the main test. The maximum exercise load test was conducted at $70 \%$ of HRmax using the Modified Bruce Protocol in order to individually set the exercise intensity to HRmax of $70 \%$. In the treadmill exercise load test, the heart rate was measured using an electrocardiogram (ECG) in real time, and the blood pressure (BP) was recorded by an automatic sphygmomanometer with a BP cuff applied to the arm, and set to automatically measure BP at 3-minute intervals. In the recovery period after finishing the treadmill exercise at $70 \%$ of HRmax, BP was measured and recorded at intervals of 2 minutes. The aerobic exercise program was performed on a treadmill. All the subjects performed exercise for 30 minutes using a treadmill load speed and gradient corresponding to HRmax of $50 \%$. Blood was collected when a stable condition, had been established and the subjects were prohibited from taking any food, smoke, drinks, or hard exercise from 10 o'clock on the night before the test day. Approximately $5 \mathrm{ml}$ of blood were taken from the antecubital vein.

The aerobic-exercise method of this study was determined based on exercise prescriptions, of intensity, frequency and time of exercise suggested by ACSM (2009), after implementing an exercise load test. The type of exercise was walking at a quick pace, and the intensity of exercise was low intensity, long-term exercise, rather than high intensity, short-term exercise, with an HRmax of $40-60 \%$. The intensity of exercise was watched by using the Polar heart rate checker in order to check the subject's exercise intensity.

The exercise was performed 5 times per week, and the exercise program was composed of 5 min warm up, 30$40 \mathrm{~min}$ main exercise and $5 \mathrm{~min}$ cool down. The control group did no exercise during the experimental period.

Statistical analyses were performed using SPSS Ver. 18.0 for Windows. ANCOVA was utilized to test the significance of differences in the variables between before and after the exercise program lasting 10 weeks. Significance was accepted for values of $\mathrm{p}<0.05$.

\section{RESULTS}

The blood lipid values and statistics of the exercise group and the control group are presented in Table 2.

Total cholesterol of the exercise group was $195.5 \pm 7.4 \mathrm{mg} /$ $\mathrm{dl}$ before the intervention and $165.9 \pm 8.3 \mathrm{mg} / \mathrm{dl}$ after the intervention. The difference between the two groups was statistically significant $(p<0.05)$. Total cholesterol of the control group was $201.9 \pm 9.3 \mathrm{mg} / \mathrm{dl}$ before the intervention and $185.20 \pm 6.7 \mathrm{mg} / \mathrm{dl}$ after the intervention. Triglyceride of the exercise group was $157.3 \pm 6.9 \mathrm{mg} / \mathrm{dl}$ before the intervention and $135.9 \pm 9.2 \mathrm{mg} / \mathrm{dl}$ after the intervention. The difference between two groups was statistically signficant $(p<0.05)$. Triglyceride of control group was $154.9 \pm 8.2 \mathrm{mg} / \mathrm{dl}$ before the intervention $158.7 \pm 9.1 \mathrm{mg} / \mathrm{dl}$ after the intervention (Table 3).

\section{DISCUSSION}

It is considered that a study to develop an appropriate program in which thorough diet control and exercise have complementary roles needs to be conducted. There are many studies which have established the relation between exercise and blood lipid levels ${ }^{1,9-11)}$. In particular, obesity is known to show a higher risk of lipid metabolism disorder than normal. Cholesterol is one of the lipids, and is widely used as a index for verifying, treating and determining hyperlipidemia $^{12)}$. This study which was conducted to measure, TC, and it showed a significant improve in the exercise group, whereas the control group displayed a non-significant. An aerobic program for women with obesity was con- 
Table 2. Changes in TC (Unit: $\mathrm{mg} / \mathrm{dl}$ )

\begin{tabular}{lcc}
\hline \multirow{2}{*}{ Variable } & \multicolumn{2}{c}{$\mathrm{TC}(\mathrm{mg} / \mathrm{dl})$} \\
\cline { 2 - 3 } & pre & post \\
\hline Exercise & $195.5 \pm 7.4$ & $165.9 \pm 8.3^{*}$ \\
Control & $201.9 \pm 9.3$ & $185.20 \pm 6.7$ \\
\hline
\end{tabular}

Table 3. Changes in TG (Unit: $\mathrm{mg} / \mathrm{dl})$

\begin{tabular}{lcc}
\hline \multirow{2}{*}{ Variable } & \multicolumn{2}{c}{$\mathrm{TG}(\mathrm{mg} / \mathrm{dl})$} \\
\cline { 2 - 3 } & pre & post \\
\hline Exercise & $157.3 \pm 6.9$ & $135.9 \pm 9.2^{*}$ \\
Control & $154.9 \pm 8.2$ & $158.7 \pm 9.1$ \\
\hline
\end{tabular}

Values are mean \pm SD. *Significantly different between pre- and post-test.

ducted at the maximum heart rate of $60-80 \%, 5$ times a week for 50 minutes, and TC and TG showed a significant decrease ${ }^{13)}$. TC and TG are the most common lipids, covering $98-99 \%$ of all naturally occurring lipids in nature. They are mainly stored in the liver and subcutaneous fat; is produced in fat tissues and the liver. The TG which is stored in fat tissues is rapidly metabolized when sugar runs short as energy source. Exercises were reported to facilitate fat utilization rate in exercise by increasing mitochondria in the skeletal muscle, promoting oxidation ability, and inhibiting the use of glycogen. It reduces body fat by decreasing TG and activating catabolic enzyme for lipoprotein, thus enabling fat to be used as an energy source.

In a study of exercise performed by female college students, both the group of diet therapy and aerobic exercise and the group of diet therapy and circuit weight training showed a significant decreases in $\mathrm{TG}^{2}$. Also, in an obesity treatment program, middle-aged women with obesity showed a significant decrease in TG, after performing for 4 days a week for 16 weeks to burn aerobic exercise and resistance weight training at $400 \mathrm{Kcal} / \mathrm{session}^{14)}$. In the present study, TC and TG in the exercise group significantly decreased. This result suggests that regular exercise should be performed along with an appropriately controlled diet program in order to improve blood lipid levels ${ }^{15}, 16$ ). Women in middle age facing menopause are encouraged to not only perform exercises, but also run with weight training to prevent sharp loss of muscle, increase of body fat mass caused by decrease of metabolism at rest and osteoporosis resulting from decline in bone density, with a view to health maintenance and obesity management. These days, weight training is becoming popular, and it is a highly proper training method for promoting the physical strength of men and women of all ages, and children.

\section{REFERENCES}

1) Korea Obesity Academic Society: Clinic Obesity. Seoul: Korea Medicine, 2001.

2) An MY: The Effect on Fat, TCHO, Glucose and TG of Middle-aged Women by Regular Exercise. KAHPERD, 2000, 39: 351-358.

3) Hang JC: Obesity Preventive and Perfect Cure All Branches of Knowledge. Taeeue Co., 2000.

4) Hwang WW, Kim KJ: Change of blood lipid profiles and body composition following a different set interval during weight training in obese. J Kor Exer Sci Acad, 2004, 13: 87-100.

5) ACSM: ACSM's Guidelines for Exercise Testing and Prescription by American College of Sports Medicine, 8th ed. Lippincott Williams \& Wilkins, 2009.

6) Kim SB: Effects of 8 Week Combined Exercise of Aerobics \& Circuit Weight Training on Serum Leptin, Blood Lipid Level, Heart Rate and Body Composition in Obese Women. Daejon University, 2003.

7) Boreham C, Twisk J, Savage M: Association between physical fitness and activity patterns during adolescence and cardiovascular risk factors in young adulthood. Int J Sports Med, 2002, 23: 22-26. [CrossRef]

8) Kim CK, We SD: Humen, Sport Medicine. Seoul: DaeKyung Books, 2001.

9) Byrne KP: Understanding and Managing Cholesterol: A Guide for Wellness Professionals. Campaign: Human Kinetics Books, 1991.

10) Pollock ML, Gettman LR, Milesis CA, et al.: Effects of frequency and duration of training on attrition and incidence of injury. Med Sci Sports, 1977, 9: 31-36. [Medline]

11) Choi KS: Effect of treadmill exercise programs on cardiopulmonary functions and serum lipids in adult obesity. Kor J Sports Med, 1994, 12: 246 263.

12) Lim, HJ: The effects of aerobic exercise program on body composition and blood lipids in obesity high school girls. J Kor Soc Living Environ System, 2006, 13: 189-194.

13) Km YK, Lee JY, Lee CS: Effect of 16weeks aerobic training on vascular compliance, blood pressure and basic physical fitness variables in middle aged women. Kor Soc Growth Dev, 2008, 16: 67-73.

14) Kim KL, Joung JS: The effects of obesity treatment programs on body composition, blood lipids profile and aerobic capacity in obese middle aged women. KAHPERD, 1999, 38: 440-450.

15) Kraemer WJ, Volek JS, Clark KL, et al.: Physiological adaptations to a weight-loss dietary regimen and exercise programs in women. J Appl Physiol 1985, 1997, 83: 270-279. [Medline]

16) Park RJ, Son HH, Sakamoto M, et al.: The effect of wearing shoes generating micro-currents on body composition and blood lipid concentrations of overweight remales. J Phys Ther Sci, 2011, 23: 177-180. [CrossRef] 